\title{
I trained overseas, what do I need to do to work in the UK as a doctor?
}

Clarification: This Careers article (BMJ 2020;368:m396, doi:10. 1136/bmj.m396, published 3 February 2020) has been amended to make it clear that it is aimed at doctors coming to the UK from countries outside the European Union. 